\section{The Turkish adaptation and psychometric properties of the Geriatric Anxiety Scale}

Faika Sanal Karahan, ${ }^{1}$ Erdal Hamarta, ${ }^{2}$ Ali Yavuz Karahan ${ }^{3}$

1Department of Psychological

Counseling and Guidance, Usak

University; ${ }^{2}$ Department of

Psychological Counseling and Guidance,

Necmettin Erbakan University, Konya;

3Department of Physical Medicine and

Rehabilitation, Usak University, Turkey

\begin{abstract}
The purpose of the present study is to translate and adapt the Geriatric Anxiety Scale (GAS), a 30-item self-report measure of anxiety among older adults, into Turkish and examine its basic psychometric properties. We tested the translated GAS in a sample of community-dwelling older adults in Konya, Turkey $\left(N=100 ; M_{\mathrm{age}}=71.4\right.$ years, $S D=6.5$; range $=65-88$ ) without a history of mental disorder and sufficient cognitive abilities. To assess the convergent validity, Turkish versions of the Beck Anxiety Inventory (BAI) and Geriatric Depression Scale (GDS) were administered. The mean GAS total score was $13.33(S D=11.86)$. Due to low item-total correlations $(<0.30)$, two items (items 2 and 3) were removed from the Turkish version of the GAS. The internal reliability (Cronbach's alpha) was excellent for the total score (0.91) and acceptable for the subscales (somatic $=$ $0.71 ;$ cognitive $=0.85 ;$ affective $=0.84$ ) . Regarding the convergent validity, the GAS total score was significantly and positively correlated with the total scores of the BAI $(r=0.87, \quad \mathrm{P}<0.05)$ and GDS $(r=0.57$, $\mathrm{P}<0.05)$, with large effect sizes. Implications: The newly translated Turkish version of the GAS has promising utility in an older adult Turkish sample. Future studies of this measure are warranted.
\end{abstract}

\section{Introduction}

Anxiety is an exceedingly common mental health problem globally. In Turkey, as in many other countries, it is not easily treated in older adults. ${ }^{1}$ Because the impact of anxiety in late-life is extensively being associated with significant functional limitation and impaired quality of life and, ${ }^{2}$ it is associated with reduced quality of life, increased disability and increased risk of suicide.3,4 Moreover, anxiety symptoms may have a negative impact on their psychosocial adjustment. Elderly patients with anxiety symptoms tend to show lower levels of autonomy, greater loss of visual and auditory acuity, neurotic traits, poor selfperceived health and quality of life, greater use of healthcare services, and increased risk of mortality. 5 Thus, finding ways to reduce anxiety in older people can be considered an important public health issue.

Unlike other mental and emotional problems in older adults, such as depression, dementia, and delirium, which are relatively popular research topics in Turkey, geriatric anxiety is not often assessed independently. Typically, depression and anxiety are assessed together. Moreover, associations between depression measures and corresponding indicators of anxiety are strongly demonstrated in teenagers, adults, and psychiatric patients.6,7 Recent data establish a very close connection between anxiety and depression and highlight the significance of modeling anxiety symptoms in assessment of depression.6,7 But this is insufficient for fully understanding geriatric anxiety. ${ }^{6-8}$ The need to understand geriatric anxiety is somewhat pressing - the elderly population were constituted $8.7 \%$ of the world population in 2016. The data from the Turkish Statistical Institute (2017) has shown that the proportion of the population that is elderly (those aged 65 years or over) has been increasing in recent decades. ${ }^{9} \mathrm{It}$ is estimated that it will be 8.6 million in 2023, which will then increase to 19.5 million in 2050 and 24.7 million in 2075; these represent proportions of the total population of $10.2 \%$ in $2023,20.8 \%$ in 2050 , and $27.7 \%$ in 2075.9 The life expectancy has become 78 years, for men 75.3 years and for women 80.7 years in Turkey in 2017. The elderly population ratio in Turkey increased by $17.1 \%$ in the last five years. ${ }^{9}$ In 2012 , while the elderly population was 5 million 682 thousand 3 people, this population has been growing by $17.1 \%$ in the last five years, in 2017 it was 6 million 651 thousand 503 people. With this growing elderly population, the need for a measure of geriatric anxiety that is effective, self-reported, widely used, easy to use, and based on the latest research is necessary for assessing geriatric anxiety disorders. One such measure could be the Geriatric Anxiety Scale (GAS). The GAS is a 30 -item self-report scale used to measure anxiety symptoms specifically among older adults, ${ }^{10}$ it comprises three subscales corresponding to the common
Correspondence: Ali Yavuz Karahan, Medical Faculty of Usak University, Department of Physical Medicine and Rehabilitation, Usak, Turkey.

Tel.: + 90.538.692.1934

E-mail: ayk222@hotmail.com, ali.karahan@usak.edu.tr.

Key words: Geriatric Anxiety Scale, psychometric properties, Turkish version.

Acknowledgements: the authors gratefully thank Dr. Segal (dsegal@uccs.edu) from Department of Psychology, University of Colorado at Colorado Springs for his support in preparing the data. The Turkish GAS is available for free for research purposes and may be obtained from Faika Sanal Karahan.

Contributions: FSK, EH, AYK, study conception and design; FSK, acquisition of data; FSK, AYK, analysis and interpretation of data; FSK, EH, AYK, drafting of manuscript: FSK, $\mathrm{EH}, \mathrm{AYK}$, critical revisions.

Conflict of interest: the authors declare no potential conflict of interest.

Conference presentation: this study was presented as a poster at The Gerontological Society of America's 67th Annual Scientific Meeting, in Washington, DC on November 59, 2014.

Funding: none.

Received for publication: 15 January 2018. Revision received: 19 March 2018.

Accepted for publication: 20 March 2018 .

This work is licensed under a Creative Commons Attribution-NonCommercial 4.0 International License (CC BY-NC 4.0).

(C) Copyright F.S. Karahan et al., 2018

Licensee PAGEPress, Italy

Mental Illness 2018; 10:7580

doi:10.4081/mi.2018.7580

anxiety symptoms among elderly people (i.e., somatic, cognitive, and affective symptoms, each with between 8 and 9 items). ${ }^{10}$ Yochim et al. ${ }^{11}$ demonstrated that the GAS is a useful multidimensional measure of anxiety among elderly adults. We considered the GAS a suitable tool for assessing geriatric anxiety on a wide scale, given that it is accessible, has been translated into various languages (e.g., Persian and German), and assesses numerous dimensions of anxiety symptoms. The purpose of this study was to translate the GAS into Turkish and verify its psychometric properties. 


\section{Materials and Methods}

\section{Translation of the Geriatric Anxiety Scale}

Two of five associates from the Department of English Language Teaching at our affiliated university translated the English version of the GAS into Turkish. The Turkish version was then back-translated into English separately by two different associates. Then, all four of these translators compared the Turkish and English versions to determine whether the items had comparable meanings. Finally, five associates from the Department of Psychological Counseling and Guidance looked over the Turkish version; based on their comments, we made a few minor changes to it. Then, to determine whether elderly adults can easily understand the scale, twenty elderly adults were asked to read over and check the comprehensibility of the GAS items. This showed that the measure was understandable and culturally suitable.

\section{Study design and participants}

This was a single-center cross-sectional study conducted in Konya, Turkey in January 2014. The study protocol was approved by the local ethics committee and all participants provided their written informed consent. Participants were recruited via advertisements or through referrals from primary care physicians. We consecutively evaluated whether 200 elderly adults met the criteria for participation. The inclusion criteria were: (a) aged $\geq 65$ years and (b) volunteered to participation. The exclusion criteria were (a) having difficulty in communicating in Turkish, (b) unable to understand the content of the questionnaires; (c) have serious cardiopulmonary dysfunction (e.g., congestive heart failure, unstable angina pectoris, third-stage cardiac block), cancer, or any other life-threatening diseases; and (d) have central or peripheral neurological disease. After applying these criteria, we administered the scales to a total of 183 participants. Data of 13 participants were ultimately excluded because they were incomplete; thus, a total of 170 participants were evaluated. Participants' ages ranged from 65-94 years $\left(M_{\mathrm{age}}=70.38\right.$, $S D=9.49)$. There were 81 men $(48 \%)$ and 89 women $(52 \%)$. One hundred two participants reported living with a spouse $(60 \%), 39$ with their children $(23 \%)$, and 29 alone or at an elderly care home (17\%). Regarding their education levels, 85 participants had a primary/secondary school education $(50 \%), 19$ had graduated from high school (11.2\%), 7 had graduated from university $(4.1 \%), 21$ were literate but had no formal education (12.4\%), and 38 were neither literate nor formally educated $(22.4 \%)$ (Table 1$)$.

Table 1. Sociodemographic profile of study participants.

\begin{tabular}{lc} 
& Total sample, $\mathbf{n}=\mathbf{1 7 0}$ \\
Age mean (SD) & $70.38 \pm 9.49$ \\
Gender, $\mathrm{n}(\%)$ & $81(47.6)$ \\
Male & $89(52.3)$ \\
Female & $28.35 \pm 4.6$ \\
\hline Body mass index mean (SD) $\left(\mathrm{kg} / \mathrm{m}^{2}\right)$ & \\
Education, $\mathrm{n}(\%)$ & $59(34.7)$ \\
None & $85(50.0)$ \\
Elementary and secondary school & $19(11.1)$ \\
High school & $7(4.1)$ \\
University degree & \\
\hline Marital status, $n$ (\%) & $102(60.0)$ \\
Married & $56(32.9)$ \\
Widowed & $12(7.0)$ \\
Separated/divorced & \\
Social and family support, $\mathrm{n}(\%)$ & $102(60.0)$ \\
Living with spouse & $39(22.9)$ \\
Living with his/her child & $20(11.7)$ \\
Living in an elderly care home & $9(5.2)$ \\
Living alone &
\end{tabular}

Table 2. Factor Loadings of the Geriatric Anxiety Scale.

\begin{tabular}{lccc} 
& Somatic & Cognitive & Affective \\
GAS13 & 0.859 & 0.305 & 0.312 \\
\hline GAS11 & 0.857 & 0.466 & 0.482 \\
\hline GAS15 & 0.853 & 0.353 & 0.319 \\
GAS7 & 0.835 & 0.385 & 0.342 \\
\hline GAS6 & 0.821 & 0.412 & 0.426 \\
GAS10 & 0.800 & 0.387 & 0.375 \\
\hline GAS14 & 0.784 & 0.420 & 0.412 \\
\hline GAS20 & 0.710 & 0.367 & 0.373 \\
\hline GAS24 & 0.305 & 0.864 & 0.398 \\
GAS25 & 0.466 & 0.863 & 0.415 \\
\hline GAS16 & 0.353 & 0.855 & 0.364 \\
GAS19 & 0.385 & 0.786 & 0.412 \\
\hline GAS5 & 0.412 & 0.768 & 0.352 \\
GAS18 & 0.387 & 0.710 & 0.403 \\
\hline GAS4 & 0.461 & 0.700 & 0.481 \\
GAS12 & 0.309 & 0.553 & 0.367 \\
\hline GAS1 & 0.307 & 0.422 & 0.837 \\
GAS21 & 0.302 & 0.502 & 0.827 \\
\hline GAS2 & 0.420 & 0.465 & 0.827 \\
GAS23 & 0.350 & 0.321 & 0.805 \\
\hline GAS22 & 0.409 & 0.497 & 0.801 \\
GAS9 & 0.476 & 0.507 & 0.761 \\
\hline GAS8 & 0.359 & 0.407 & 0.637 \\
GAS3 & 0.461 & 0.367 & 0.636 \\
\hline GAS17 & 0.303 & 0.312 & 0.412 \\
\hline \%65 & $\% 24$ & $\% 21$ & \\
\hline & & & 020 \\
\hline
\end{tabular}


frequency of symptoms in the past week; the scale ranged from 0 (not at all) to 3 (all the time). The total score of the GAS is calculated using only the first 25 items. The remaining 5 items are not included as part of the GAS score and are to be used clinically. Higher total scores indicated greater levels of anxiety. In the original validation study, the internal consistency of the GAS was excellent for the entire scale and for each subscale (total scale, Cronbach's $\alpha=0.93$; cognitive, somatic, and affective subscales, Cronbach's $\alpha=0.90,0.80$ and 0.82 , respectively). ${ }^{10}$ The convergent validity has also been confirmed - namely, the GAS has strong positive relationships with other measures of anxiety. 12

\section{Geriatric Depression Scale}

The Geriatric Depression Scale (GDS) is a widely used self-report measurement of depression in older adults. This scale was developed by Yesavage et al. ${ }^{13}$ and contains 30 items, each of which is rated dichotomously (yes or no). Items are scored as either 0 or 1 points and the total score is rated on a scoring grid. Participants with scores of 0-9 are classified as normal, those with scores of 10-19 as mildly depressed, and those with scores of 20-30 as severely depressed. ${ }^{13}$ Furthermore, the validity and reliability of the Turkish version have been confirmed. ${ }^{14,15}$

\section{Beck Anxiety Inventory}

The Beck Anxiety Inventory (BAI) is a 21-itemself-report measure of the common symptoms of clinical anxiety disorders, such as nervousness and fear of losing control. The inventory was developed by Beck et al. ${ }^{16}$ Each item is rated on a 4-point scale reflecting the degree to which each symptom disturbs participants; the answer options range from 0 (not at all) to 3 (severely, I could barely stand it). Total scores can range from 0 to 63 , with higher scores indicating more severe anxiety. ${ }^{12}$ The Turkish version of this inventory was devised by Ulusoy et al. ${ }^{17}$ The psychometric properties of this inventory were appropriate.

\section{Results}

\section{Reliability}

The internal consistency was evaluated using Cronbach's alpha coefficient. For the GAS as a whole, the Cronbach's $\alpha$ was 0.91 , which indicated excellent internal consistency. For the somatic, cognitive, and affective subscales, the Cronbach's $\alpha$ were as $0.87,0.91$, and 0.94 , respectively. These coefficients all indicate that the internal consistency was sufficient (Table 2).

\section{Content and construct validity}

The mean total score of the GAS was $18.01(S D=12.78)$. Ceiling and floor effects observed for all subscale scores and the total score were all at the acceptable level $(<5 \%)$, indicating appropriate content validity.

To determine whether the sample met the assumptions for factor analysis, which was used to measure the construct validity, we calculated the Kaiser-Meyer-Olkin measure of sampling adequacy (KMO value) and performed Bartlett's test of sphericity. The KMO value was 0.88 , while the Bartlett's test results were significant $\left(\chi^{2}=3317.156, \mathrm{P}<0.001\right)$. These indicated that the sample was suitable for factor analysis. Table 2 shows the factor loadings of the 25 GAS items. The results of a principle component analysis with an oblique rotation yielded three factors, which is consistent with the original GAS. The factors were labeled cognitive, somatic, and affec- tive symptoms and together described $65 \%$ of the variance. More specifically, the somatic subscale explained $24 \%$ of the total variance and had factor loadings ranging from 0.71 to 0.85 . The cognitive subscale explained $21 \%$ of the total variance and its factor loadings ranged from 0.55 to 0.86 . Finally, the affective subscale explains $20 \%$ of the total variance and its factor loadings ranged from 0.41 to 0.83 . A scree plot of the factor analysis results is shown in Figure 1.

The correlations between the subscales of the GAS are as follows. First, we found that the somatic subscale had a positive, significant, and moderate relation with the cognitive subscale $(r=0.588)$ (Table 3$)$. There was also a positive, significant, and moderate correlation between the somatic and affective subscales $(r=0.581)$, and a positive, significant, and strong correlation between the cognitive and affective subscales $(r=0,783)$ (Table 3).

\section{Convergent validity}

The results of a correlation analysis showed that the total and subscale scores of

Table 3. Correlations between subscales of the Geriatric Anxiety Scale.

\begin{tabular}{lcccc} 
& n:170 & Somatic & Cognitive & Affective \\
Somatic & $r$ & 1.000 & $0.588^{*}$ & $0.581^{*}$ \\
Cognitive & $r$ & $0.588^{*}$ & 1.000 & $0.783^{*}$ \\
\hline Affective & $r$ & $0.581^{*}$ & $0.783^{*}$ & 1.000 \\
\hline
\end{tabular}

All tests are two-tailed. ${ }^{*} \mathrm{P}<0.01$

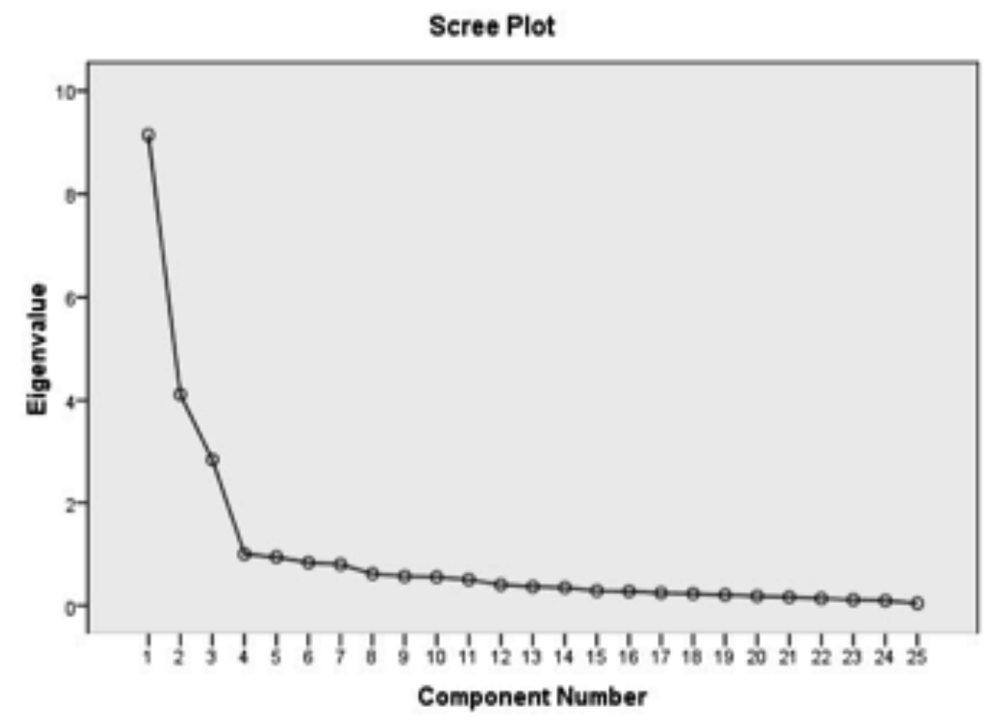

Figure 1. Scree plot of the factor analysis. The factors at the point of being shaped on the horizontal axis were accepted as the number maximum of factors. For eigenvalues, factors with eigenvalues of $>1$ were accepted, and were excluded otherwise. Four factors had eigenvalues of $>1$. While the first factor explained $16.215 \%$ of the total variance, four factors together explained $49.262 \%$. Beginning from the fourth factor, the tendency was lost in an important level. Thus, the number of factors was limited with 4 to 5 factors. 
the GAS all had significant positive correlations with the GDS and BAI (Table 4). More specifically, there was a weak relationship (albeit still significant) between the somatic subscale and the GDS total $(r=0.456)$, and a moderate correlation between this subscale and the BAI total $(r=0.662)$. The cognitive subscale had a moderate correlation with the GDS $(r=0.601)$ and a strong one with the BAI $(r=0.830)$. The affective subscale had a moderate correlation with the GDS $(r=0.557)$ and a strong correlation with the BAI $(r=0.755)$. Finally, the GAS total score had a moderate correlation with the GDS $(r=0.608)$ and a strong correlation with the BAI ( $r=0.849)$ (Table 4).

\section{Discussion}

The older adult population in Turkey is growing. The percentage of older adults was $5 \%$ in 2000 but is expected to reach $23 \%$ by 2050.9 With the increased older adult population comes a greater need for valid and brief mental health screenings and assessment in Turkish. The purposes of this study were to translate the Geriatric Anxiety Scale into Turkish, and to initially evaluate the psychometric properties of the new measure in a large sample of communitydwelling older Turkish adults. Anxiety is an important health problem among older adults. ${ }^{6}$ We thus adapted a highly accessible, easy-to-use measure of anxiety specifically targeting elderly adults for use in Turkey. In this study authors administered the questionnaire to a sample of community dwelling Turkish older adults and the results of the study revealed that the Turkish version of the GAS is promising. Nevertheless, further research is likely needed to confirm the psychometric properties of the Turkish GAS in different samples of the Turkish elderly population, such as with psychiatric problems.

The concurrent validity and internal consistency of obtained for the Turkish version of the scale were coherent and like those obtained for the original version. ${ }^{10} \mathrm{In}$ the original validation study, the internal consistency of the GAS was excellent for the entire scale and for each subscale (total scale, Cronbach's $\alpha=0.93$; cognitive, somatic, and affective subscales, Cronbach's $\alpha=0.90,0.80$ and 0.82 , respectively). ${ }^{10}$ In the reliability analyses, two items of the Turkish version (items 2 and 3) were removed due to low (below 0.3) itemtotal correlation coefficients. All 23 items had item-total correlations of 0.412 or more and most items were above 0.7 . Concurrent validity between the Turkish GAS and the other two measures of anxiety: BAI and GDS was assessed with Pearson productmoment correlations. The results of correlation coefficient show that Turkish GAS is an effective instrument to measure anxiety in elderly people.

Regarding item properties, the featured items clearly differed in terms of threshold parameters. The GAS was successfully translated into Turkish through the doubleblind method. Reliability estimates (Cronbach's $\alpha$ ) of the new measure were all adequate and similar to reliability data for the English version. ${ }^{10}$ Findings also revealed that the Turkish GAS has adequate convergent validity as compared to the BAI and to a lesser degree, the GDS. Potential advantages of the GAS are that it was designed specifically for older adults and that it provides evaluation of the overall levels of anxiety as well as an assessment of three important facets of anxiety (cognitive, somatic, and affective dimensions).

In Turkish version, the mean total score of the GAS was $18.01(S D=12.78)$. This pattern is in accordance with the Iranian sample (mean score $=18.94, S D=12.65$ ). The low means in the US sample10 (mean score $=13.65, S D=9.70)$, Italian sample 18 (mean score $=13.08, S D=7.95)$ and the German sample ${ }^{19}$ (mean score $=10.51$, $S D=8.95$ ) were reported. According to this result, it becomes clear that the mean scores in western societies samples were considerably lower than in the eastern samples. This result is in accordance with the higher prevalence of anxiety among Turkish population $(18 \%)$, compared to adults in US (between 3 and 14\%) or the Germany (4.5\%).
There were some important drawbacks to this study. The main limitation is the sample was composed solely of non-clinical elderly people living in community. So, the findings may not be generalizing to other groups of elderly adults, such as psychiatric patients. Additionally, we know anxiety and depression are more closely related than they seem. However, this study does not include assessment of discriminant validity. Thus, following studies should further analyze the Turkish version of the GAS. Although the preliminary results in this study are promising, further research should explore the psychometrics of the Turkish GAS in larger and more diverse samples of Turkish older adults, including psychiatrically impaired older adults.

\section{Conclusions}

In summary, the Turkish version of the GAS appears to have has promising utility in an older adult Turkish sample, with adequate internal scale reliability and convergent validity. The findings of present study provide promising evidence for reliability of the Turkish-GAS, its discriminant and convergent validity. Also, with the successful translation of the GAS, highly reliable and valid scale provided. With regard to external criteria, the Turkish-GAS by and large discriminated between cases with and without anxiety related characteristics. Future studies should explore the psychometrics of this measure in larger and more diverse samples of older adults in Turkey, including psychiatrically impaired older adults. Factor analytic studies are needed to evaluate the factor structure of the translated measure.

\section{References}

1. Bolghan-Abadi M, Segal DL, Coolidge FL, et al. Persian version of the geriatric anxiety scale: Translation and preliminary psychometric properties among Iranian older adults. Aging Ment Health 2013;17:896-900.

2. Forlani M, Morri M, Murri MB, et al. Anxiety Symptoms in $74+$ communitydwelling elderly: associations with physical morbidity, depression and alcohol consumption. PLoS One 2014;9:e89859.

3. Unützer J, Bruce ML. The elderly. Ment Health Serv Res 2002;4:245-7.

4. Titov N, Fogliati VJ, Staples LG, et al. Treating anxiety and depression in older adults: randomized controlled trial com- 
paring guided v. self-guided internetdelivered cognitive-behavioral therapy. BJ Psych Open 2016;2:50-8.

5. Fernandez-Blazquez MA, AvilaVillanueva M, Lopez-Pina JA, et al. Psychometric properties of a new short version of the State-Trait Anxiety Inventory (STAI) for the assessment of anxiety in the elderly. Neurologia 2015;30:352-8.

6. Akyol Y, Durmus D, Dogan C, et al. Quality of life and level of depressive symptoms in the geriatric population. Turk J Rheumatol 2010;25:165-73.

7. Koc Z, Saglam Z. The determination of anxiety and depression levels among hospitalized older people. Turk J Res Dev Nurs 2011;13:42-52.

8. Kilınc S, Torun F. Depression rating scales used in clinical practice in Turkey. Medical Newspaper 2011;86:39-47.

9. Turkish Statistical Institute. Population Projections, 2017-2075 (Report No: 15844). Available from http://www.turkstat.gov.tr/PreHaberBul tenleri.do? id $=15844$

10. Segal D L, June A, Payne M, et al. Development and initial validation of a self-report assessment tool for anxiety among older adults: The Geriatric
Anxiety Scale. J Anxiety Disord 2010;24:709-14.

11. Yochim BP, Mueller AE, Segal DL. Late life anxiety is associated with decreased memory and executive functioning in community dwelling older adults. J Anxiety Disord 2013;27:56775.

12. Yochim BP, Mueller AE, June A, Segal DL. Psychometric properties of the Geriatric Anxiety Scale: Comparison to the Beck Anxiety Inventory and Geriatric Anxiety Inventory. Clin Gerontol 2011;34:21-33.

13. Yesavage JA, Brink TL, Rose TL. Development and validation of geriatric depression screening scale: a preliminary report. J Psychiatr Res 1983;17:37-49.

14. Sagduyu A. The Depression Scale for the older people: a reliability and validity study compared with the Hamilton Depression Scale. Turk Psychiatr J 1997;8:3-9.

15. Ertan T, Eker E, Sar V. The validity and reliability of the Geriatric Depression Scale among the Turkish elderly population. Neuropsychiatr Arch 1997;34:62-71.

16. Beck AT, Epstein N, Brown G, Steer RA. An inventory for measuring clini- cal anxiety: psychometric properties. J Consult Clin Psychol 1988;56:893-7.

17. Ulusoy M, Sahin NH, Erkmen H. The Turkish version of the Beck Anxiety Inventory: Psychometric properties. J Cogn Pschother 1998;12:163-72.

18. Gatti A, Gottschling J, Brugnera A, et al. An investigation of the psychometric properties of the Geriatric Anxiety Scale (GAS) in an Italian sample of community-dwelling older adults. Aging Mental Health, 2017 [In press].

19. Gottschling J, Segal DL, Hausele C, et al. Assessment of anxiety in older adults: Translation and psychometric evaluation of the German version of the Geriatric Anxiety Scale (GAS). J Psychopathol Behav Assess 2016;38:136-48.

20. Wolitzky-Taylor KB, Castriotta N, Lenze EJ, et al. Anxiety disorders in older adults: a comprehensive review. Depress Anxiety 2010;27:190-211.

21. Schaub RT, Linden M. Anxiety and anxiety disorders in the old and very old - Results from the Berlin Aging Study (BASE). Comprehensive Psychiatry 2000;41:48-54. 